\title{
SNOW STRATIGRAPHY
}

\section{MEASURED BY AN ACTIVE MICROWAVE SYSTEM}

\author{
by
}

\author{
Kazuo Fujino, Gorow Wakahama
}

Institute of Low Temperature Science, Hokkaido University, Sapporo, Japan 060

\author{
Masahiro Suzukt, Tadashi Matsumoto, and Daisku Kuroiwa
}

Hokkaido Institute of Technology, Sapporo, Japan 061-24

\begin{abstract}
Snow stratigraphy was obtained in the laboratory and field, using a FM-CW radar system having microwave frequencies ranging from $2-8 \mathrm{GHz}$ and from $6-12$ GHz. A multi-layered model consisting of artificial snow such as glass beads or polystyrene plastics was used in the laboratory. In the field, the thickness of an individual layer within a snowpack was determined by analyzing the profile of responses from interfaces within the snowpacks. Large anomalies of the responses which produce misleading results, were found to have been caused mainly by multi-reflection between stratified layers as well as residual mismatch reflection in the system's components.

The physical properties of a snowpack were measured by digging a snow pit. The comparison of the microwave responses in the profile with the visual stratigraphic layering of snow was made by inserting a reflection plate between individual layers within the snowpack.
\end{abstract}

\section{INTRODUCTION}

Marked progress has recently been noted in the development of remote sensing techniques using an electromagnetic wave, in determining and monitoring depth, water equivalence and physical properties of snowpack.

One of the advantages of microwave sensing is that the method does not disturb the snowpack which is being investigated. Microwave sensors are potentially capable of meeting such a practical demand only if they make it posssible to provide a correct understanding of a complicated interrelationship among the various physical properties and their effects on the responses from the interface in the snowpack. A lack of this understanding has made somewhat ambiguous the interpretation of the data obtained concerning the physical parameters (Ulaby 1982, Matsumoto 1983).

Snowpack has a multi-layered structure, and the components of each layer change with time from freshfallen to coarse granular snow. The dielectric properties which control behavior and response to microwaves also change the physical parameters in the snowpack.

An FM-CW active microwave system has the characteristic of detecting reflection from the interfaces within the medium, and enables us to obtain the record of a profile through the medium. Thus, this system is considered fairly effective in remotely measuring and monitoring stratigraphic features within the snowpack. Consequently both theoretical and experimental investigations have been made for several years (Boyne 1980). Experimental applications have also been made to the snowpack, and the data obtained have been interpreted in terms of such physical properties as stratigraphy, density and free water content. But still many ambiguous points remain in the interpretation of the data.

This paper reports on measurements of stratigraphy within a snowpack with an FM-CW system having bandwidths of 2-8 GHz and 6-12 GHz. Also presented are test results, interpreted in terms of physical parameters such as density and free water content.

\section{MEASURING SYSTEM}

The block diagram of the FM-CW radar system developed and used for our experiments is given in Figure 1. Emitting power is about $40 \mathrm{~mW}$; and microwave frequencies ranging from $2-8 \mathrm{GHz}$ and from $6-12 \mathrm{GHz}$ are used, sweeping continuously over the badnwidth every

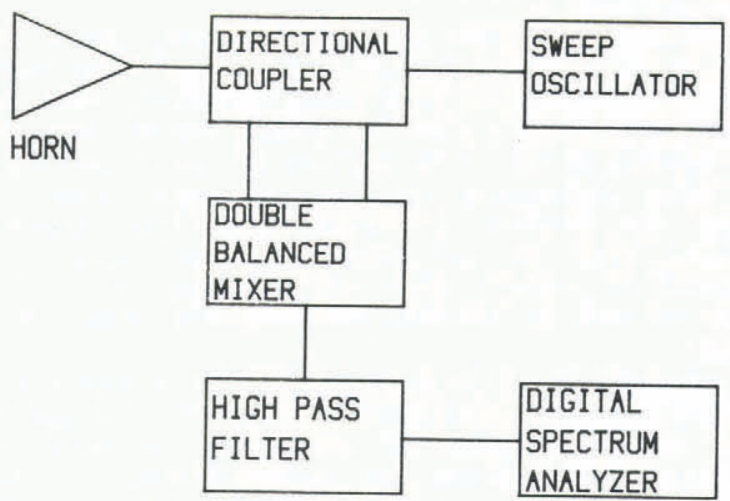

Fig.1. Block diagram of the FM-CW radar system used for measurements.

10 to $100 \mathrm{~ms}$. A single antenna set at a near-normal incident with respect to the snowpack surface emits and receives signals. Data collected in the time domain are processed with a Fast Fourier Transformer to the frequency domain. The location of the spectra displayed are functions of the distance from the antenna to each interface and their dielectric constant. They are presented as

$$
d=c \Delta f /(2 \sqrt{\epsilon} d f / d t)
$$

where $d$ is a difference in distance between two interfaces and $c$ the speed of light, $\epsilon$ the dielectric constant of snowpack relative to air, and $\mathrm{df} / \mathrm{dt}$ the change of frequency of the system per unit time.

\section{EXPERIMENTAL RESULTS}

A snowpack has a multi-layered structure. Each layer consists of ice grains of different size, shape and configuration. Liquid water is an additional consideration in the melting season.

The complex dielectric properties of the snowpack are a function of these parameters. Even in dry snowpack the relative dielectric constant changes from nearly 1 for fresh fallen snow to about 2 for coarse granular snow (Stiles 1981).

Meanwhile, the thickness of each layer within it, 
which is measured by the system, is equivalent to the electrical path length, which is a function of the actual length and the dielectric constant. Therefore, without assuming the proper value of relative dielectric constant for the snowpack or each layer, actual depth or thickness of them cannot be obtained, in the same way as for the exact relative dielectric constant.

For our purpose, it is necessary to find the proper values of relative dielectric constant for the typical snowpack, either dry or wet, as basic information to analyze the spectra obtained by the system.

\section{Laboratory experiments}

Laboratory experiments were carried out using simple-layered models of artificial snow (glass beads, and foamed polystyrene plastics) and natural wet snow.

Firstly, the relations of the thickness of each layer with the locations of individual spectra from the surface, the bottom and the interface within the model were obtained with respect to size and compactness together with the systems parameters selected such as frequency bandwidth, sweep time of oscillator and filter ranges.

From the results obtained for the dry snowpack model, the following were confirmed: (1) the configuration and apparent density of a layer had a considerable effect on the relative dielectric constant of the layer; (2) the whole thickness of the model was obtainable with a considerable degree of accuracy assuming the average value of relative dielectric constant for the model; (3) reflection due to mismatch of the system's components and multi-reflection at the interface of air-glass beads were so large that they precluded acccurate interpretation of the spectra.

Secondly, foamed polystyrene plastic plates were piled up on the reflection plate; and, on the top surface of it, several sheets of filter paper containing water were placed, and also a metal net having various mesh sizes was inserted parallel with surfaces at several portions of the block substituting for the wet layers. Using this model, the locations of spectra and their intensities reflected from at the interfaces were measured by changing the volume of water contained in the filter paper, as well as changing the frequency ranges of the system.

One of the results obtained is given in Figure 2 and the following were confirmed: the intensity of a spectrum from the top surface of the block increased with increasing water volume but saturated when the water volume exceeded a certain volume; and remarkable masking of intensities of spectra from underlying interfaces within the block was observed with increasing water volume of the surface layer.

Thirdly, a snow block of homogeneous structure was placed on the plastic block and kept at room temperature of $25^{\circ} \mathrm{C}$. The same measurement as the second experiment was made as a function of time changing with melting of the snow block.

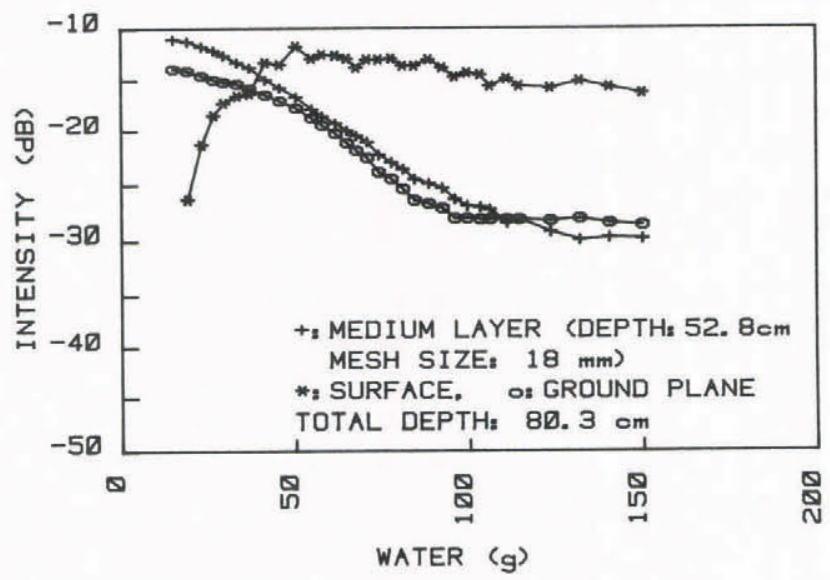

Fig.2. Relation between water volume contained in filter paper on the surface and intensity of spectra from the interfaces.

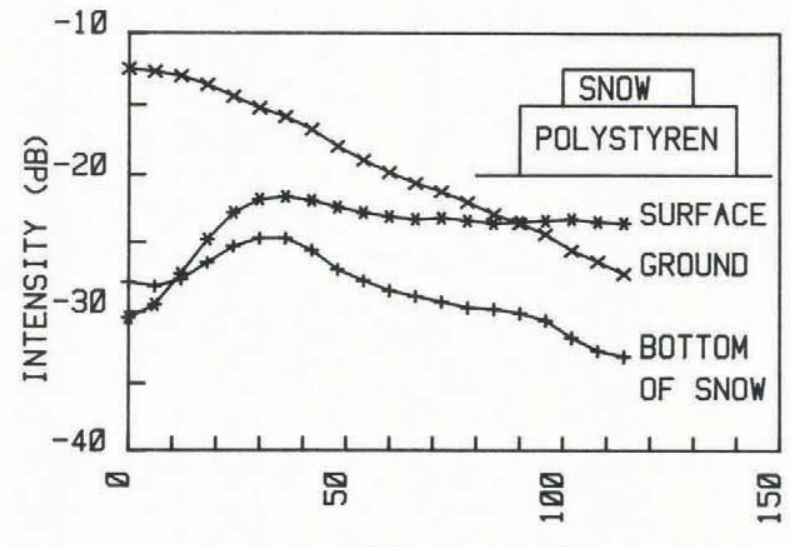

TIME (minute)

Fig.3. Variation with time in intensity of spectra from each interface.

One of the results obtained is given in Figure 3. The same tendencies as the second experiment were confirmed. Remarkable changes in intensity which were expected were not observed in either experiments with changes in the system's parameters.

\section{Field experiments}

Field experiments have been carried out in northern Hokkaido since 1982, where January and February ambient temperatures are usually below $0^{\circ} \mathrm{C}$. The snowpack in this area remains dry during these months, but becomes wet by melting in March and April when temperatures rise above freezing point.

A metal net having a mesh of $1 \mathrm{~cm}$ was placed on the ground as a reflection plate before the snow season, and a tower was set above the metal net. Total snow depth at the site was $60-120 \mathrm{~cm}$ during experiments Measurements were made at the site of the tower at least once a month, through the snow season.

Stratigraphy and the profile of physical parameters such as grain size, density and temperature throughout the snowpack were measured at the same time as the experiments by digging a pit near the tower. One of the typical structures of the snowpack in the dry-snow season is given in Figure 4.

In initial stages of the experiments it was difficult to identify the spectra, corresponding to the interfaces within the snowpack, so a reflection plate was inserted into each visible interface from the pit wall; significant spectra were then confirmed, together with the actual depths of the interfaces.

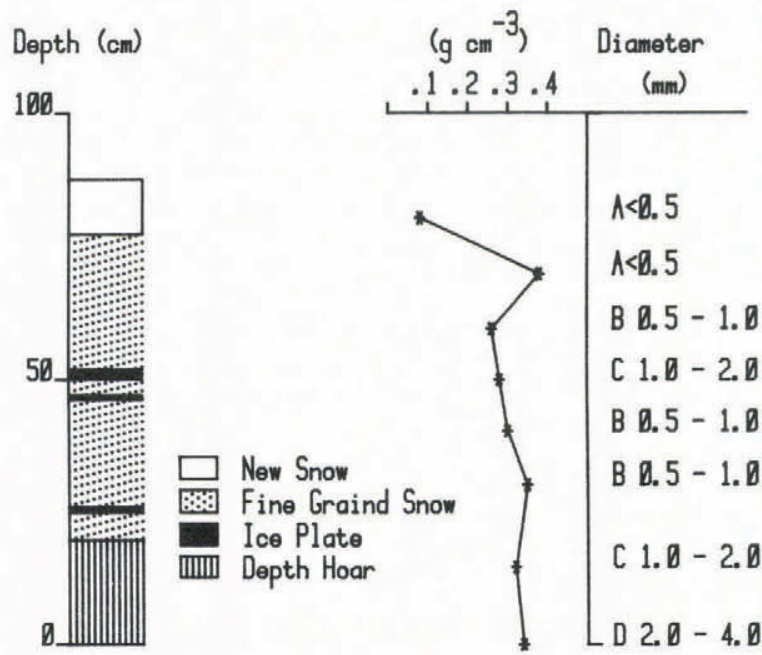

Fig.4. Typical structure of a dry snowpack. 


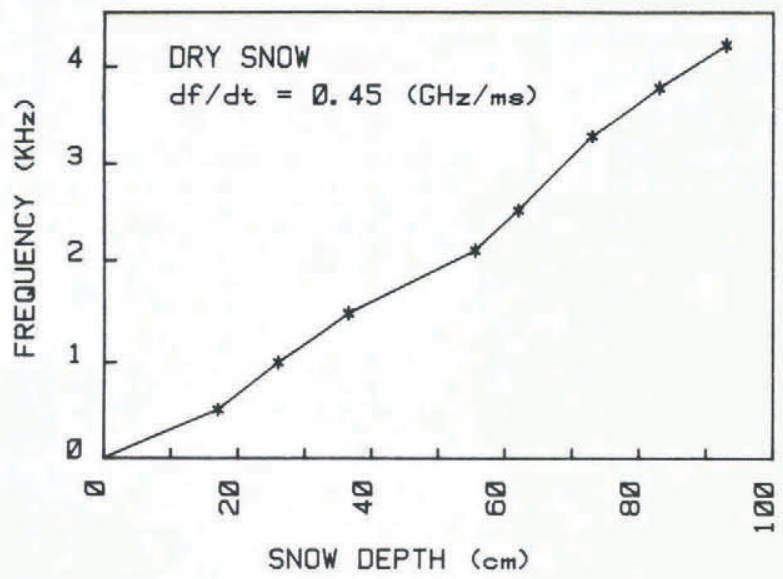

Fig.5. Variation in frequency with snow depth examined by inserting a reflection plate.

Figure 5 shows the relation between the actual depth of each interface where a reflection plate was inserted, and the corresponding frequency difference of each spectrum. The snowpack stratigraphy is the same as that given in Figure 4.

In the figure, the difference in gradient of a line connecting each point expresses a difference in relative dielectric constant of each layer. From the comparison between Figures 4 and 5, a similarity can be seen between the profile of density and that of relative dielectric constant.

The average value of relative dielectric constant can be estimated to be about 1.66 from the frequency difference between the top and the bottom and the actual depth of the snowpack.

Continual observations of the stratigraphy of the snowpack were made every 30 minutes beginning at 08:00 on the morning of 2 February 1984; and at the same time measurements were made also for physical parameters of the snowpack. It had been snowing from the previous evening till the morning and the snowpack depth increased to $105 \mathrm{~cm}$ that morning.

Spectra obtained at 09:00 are given in Figure 6. In the figure, spectra corresponding to the top and the

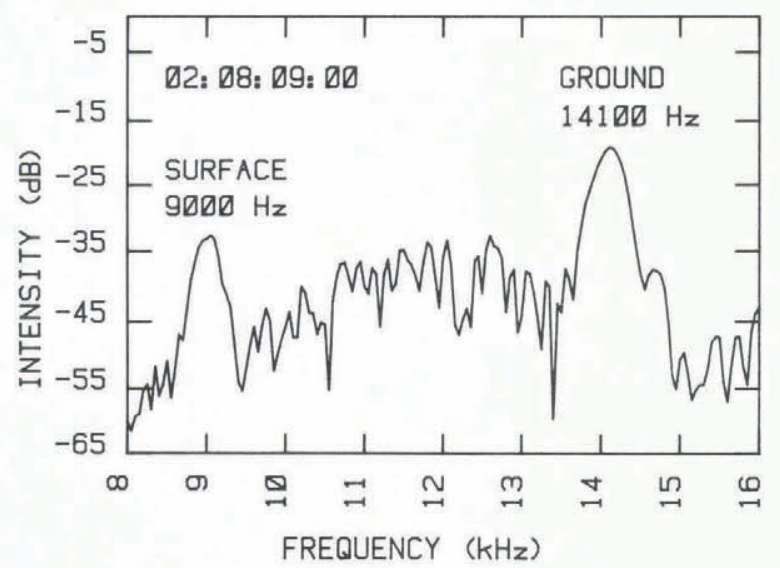

Fig.6. Profile of spectra of the whole snowpack obtained at 09:00 on 2 February 1984.

bottom surface can be clearly recognized; frequency difference and actual depth can be measured. For this measurement, $\Delta \mathrm{f}=5,100 \mathrm{~Hz}, \mathrm{df} / \mathrm{dt}=0.6 \mathrm{GHz} / \mathrm{ms}$ and $\mathrm{d}=$ $105 \mathrm{~cm}$; so we obtain $\epsilon_{\mathrm{T}}=1.47$ as the average value of relative dielectric constant of the snowpack.

Spectra obtained at 18:00 on the same day are given in Figure 7. Recognizable changes of spectra corresponding to the surface and the interfaces within the snowpack are observed in shape, intensity and location. The difference in frequency obtained at the surface

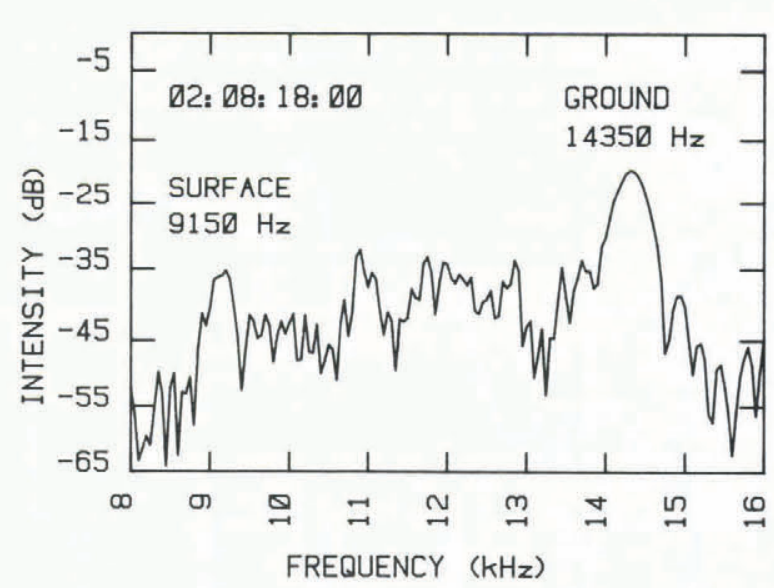

Fig.7. Profile of spectra of the same snowpack as given in Fig.6, obtained at 18:00 on 2 February 1984.

between $08: 00$ and $18: 00$ was $\Delta f=150 \mathrm{~Hz}$. The relative dielectric constant of air is $\epsilon \mathrm{a}=1$, therefore we can obtain $\mathrm{d}=3.8 \mathrm{~cm}$ as the actual depression of the air-snow interface, and this value corresponds to a decrease in actual depth of the snowpack. Also, the location of the spectrum corresponding to the bottom changes, so that we can estimate the average relative dielectric constant of the snowpack as $\epsilon_{\mathrm{T}}=1.67$, by putting $\Delta f=5,200 \mathrm{~Hz}, \mathrm{~d}=101.2 \mathrm{~cm}$.

During that time span, the surface did not melt; so these changes were caused mainly by the densification of fresh fallen snow within the upper layer of the snowpack.

By the end of March, ambient temperature of this area usually rises above $0{ }^{\circ} \mathrm{C}$ in the daytime, thereby causing the entire snowpack to become wet. Spectra obtained from the wet snowpack on 4 April 1984 are given in Figure 8. The spectrum corresponding to the surface can be recognized but that corresponding to the bottom cannot be identified, although the same metal net as in the dry snowpack was placed on the interface.

Then, by inserting a reflection plate into the snowpack, measurements were made of the detectable distance of the system for the wet snowpack, changing the distance from the surface to the plate. As a result, the detectable distance of the system was found to be less thatn $20 \mathrm{~cm}$, depending on the free water content of the layer.

It can be understood that an increase in free water content in the snowpack brings about an increase in the imaginary part of the complex dielectric constant of the snowpack; consequently, the attenuation of a microwave within the snowpack increases, and the reflection from the underlying interface decreases.

The intensity of a spectrum corresponding to the air-snow interface contains a variety of information

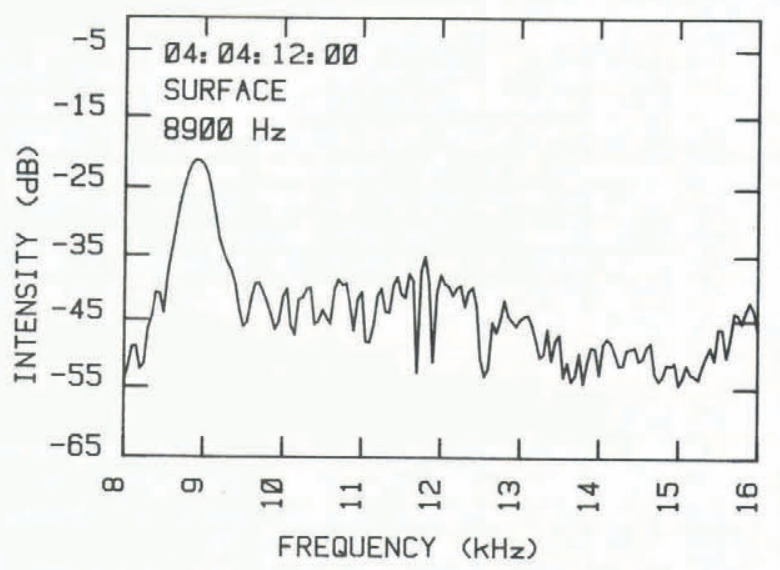

Fig.8. Profile of spectra of a wet snowpack obtained at 04:00 on 4 April 1984. 


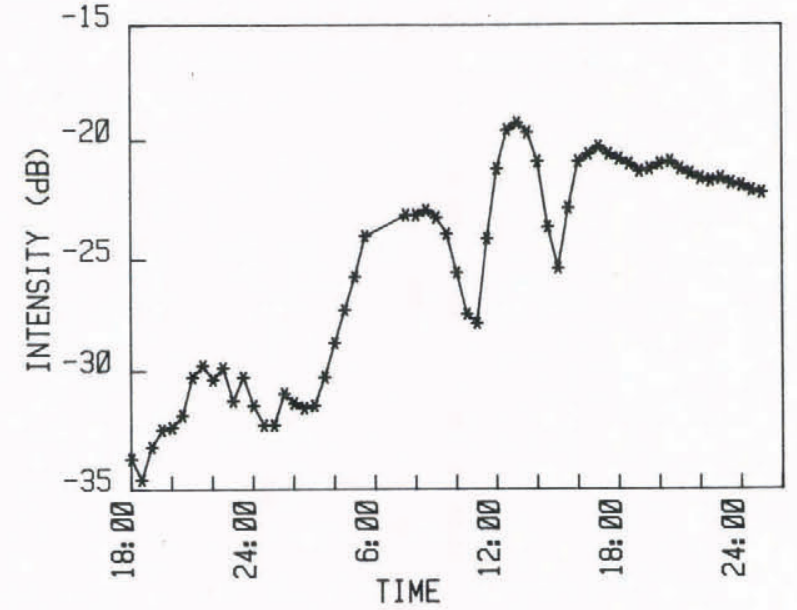

Fig.9. Variation with time in intensity of spectra from the surface.

within the uppermost layer of the snowpack such as free water content within the surface and subsurface structure.

Changes in intensity of the spectrum corresponding to the air-snow interface with time are given in Figure 9. These can be explained as follows. During the night time, from 18:00 to $04: 00$, melting of the near surface layer ceases, so that the layer is kept dry and reflection from the layer is not so large. But during day time, free water within the layer increases due to melting. When meltwater which is kept within the layer reaches the saturation level, it begins to flow down to the lower layers. The reflection from the layer decreases corresponding to a decrease in the free water content within the layer. After 18:00 of the second day, the meltwater is still kept in the near surface layer without freezing but gradually decreases due to capillary flow down to the layer below, so that the reflection from the layer is also kept quite large.

This was also confirmed from a change in free water content within the upper layer of the snowpack, which was measured by the calorimetric method during that time span.

\section{CONCLUDING REMARKS}

The stratigraphy of a snowpack has been obtained using a FM-CW radar system throughout several snow seasons. Although the results obtained demonstrate the potential use of the active microwave system for monitoring the snowpack, the following must be needed for improving it: (1) it calls for further investigations of the relation between various physical parameters of the snowpack as a function of microwave frequency; (2) it necessitates the development of theoretical models as a function of simplified snow parameters.

From the practical viewpoint the following must be taken into account: (1) for eliminating ambiguities from the analyses and increasing the accuracy of measurements, it will be useful to adopt such typical values of relative dielectric constant for the seasonal and the dry/wet snowpack; (2) for obtaining an outline of the profiles of layers and densities within the snowpack, use of the present system will be reinforced by adopting a simple method of ram sounding that can be applied with relative ease compared to the pit method.

\section{REFERENCES}

Boyne H S, Ellerbruch D A 1980 Active microwave water equivalence measurements. Proceedings of $a$ workshop on the microwave remote sensing of snowpack properties, Fort Collins, Colorado: 119-128

Matsumoto T 1983 [Recent aspects of the researches on the microwave sensing of snowpack] (in Japanese). Journal of IECE of Japan 66(3): 225-231
Stiles W H, Ulaby F T 1981 Dielectric properties of snow. Proceedings of a workshop on the properties of snow. Snow bird, Utha: 91-103

Ulaby F T 1982 Radar signatures of terrain: useful monitors of renewable resources. Proceedings of IEEE 70(12): $1410-1428$ 